\title{
Aplicación de herramientas digitales para la construcción y consolidación de aprendizajes en la asignatura Desarrollo Curricular de Ciencias Sociales I
}

\author{
Tatiana Karina Aragón Aguilar \\ Licenciada en Ciencias de la Educación con especialidad en Educación Básica \\ Docente investigadora, Facultad de Ciencias y Humanidades \\ Universidad Católica de El Salvador, El Salvador \\ Email: tatiana.aragon@catolica.edu.sv \\ ORCID: https://orcid.org/0000-0002-8671-1019
}

Fecha de recepción: $15-12-2019$

Fecha de aceptación: 21-02-2020

\section{Resumen}

La enseñanza de las Ciencias Sociales ha estado constantemente involucrada en los procesos tradicionales, monótonos, en donde el componente cognitivo-conceptual ha sido el protagonista. Por ello, es necesario introducir de manera gradual herramientas de tecnología educacional, que propicien el aprendizaje activo y significativo en los alumnos de Educación Superior, que se preparan como futuros educadores.

El propósito de la investigación-acción fue mejorar el proceso de enseñanza-aprendizaje, mediante herramientas digitales para la construcción y consolidación de aprendizajes en la asignatura Desarrollo de Ciencias Sociales I durante el ciclo I-2019. Tras el diagnóstico de la situación y el contexto que caracterizaba el proceso de enseñanza-aprendizaje de la asignatura, se establecieron técnicas didácticas basadas en el uso de herramientas digitales. Estos insumos se aplicaron en la construcción y consolidación de aprendizajes, a fin de reflexionar sobre los resultados obtenidos.

Tras la implementación de la tecnología educativa, los estudiantes mostraron un aumento significativo en la motivación durante la clase. Se evidenció la preferencia por el trabajo colaborativo, así como una mejora en los resultados cualitativos y cuantitativos dentro de su proceso de formación. Se comprobó la importancia de trabajar con las TIC, ya que favorecen el autoaprendizaje y la elaboración de productos con componentes visuales, en donde aplican la creatividad.

Palabras clave: Tecnología educacional, trabajo colaborativo, proceso de enseñanza-aprendizaje, autoaprendizaje, TIC.

\begin{abstract}
The teaching of Social Sciences has been constantly involved in traditional monotonous processes where the cognitive-conceptual component has been the protagonist. Therefore, it is necessary to gradually introduce educational technology tools that promote active and meaningful learning in Higher Education students, who prepare as future educators.

The purpose of the action research was to improve the teaching-learning process, through digital tools for the construction and consolidation of learning in the subject Development of Social Sciences I during the semester I-2019. After the diagnosis of the situation and the context that characterized the teaching-learning process of the subject, didactic techniques based on the use of digital tools were established. These inputs were applied in the construction and consolidation of learning, in order to reflect on the results obtained.

After the implementation of educational technology, students showed a significant increase in motivation during class. it was demonstrated the preference for collaborative work, as well as an improvement in the qualitative and quantitative results within their training process. It was proved the importance of working with ICTs, since they favor self-learning and the development of products with visual components, where creativity is applied.
\end{abstract}

Key words: Educational technology, collaborative work, teaching-learning process, self-learning, ICT. 


\section{Introducción}

La enseñanza de las Ciencias Sociales, en todos los niveles educativos, ha estado marcada por la sombra de la tradicionalidad y la preponderancia del componente memorístico, especialmente en las áreas correspondientes a Historia y Geografía. Tal como lo afirma Sánchez (1992):

La enseñanza del área de Ciencias Sociales es, sin duda, una cuestión problemática. Los psicólogos acusan a la enseñanza tradicional de la Historia, por ejemplo, de centrarse únicamente en la memoria. Pero, por otro lado, demuestran en sus estudios que, la Historia que se pretende enseñar, actualmente exige un pensamiento formal; que los alumnos de primaria y secundaria no poseen plenamente (p. 44).

Por otro lado, Fontana (1982), citado por Sánchez (1992), afirma que la Historia que se enseña actualmente en los centros de estudio, desde secundaria hasta a universidad, no parece dar respuesta a las necesidades y preocupaciones de los adolescentes y jóvenes (p. 44).

Esta necesidad de habilidades del pensamiento formal para comprender las diversas ramas de las Ciencias Sociales se ve reflejada en el documento oficial, Currículo al Servicio del Aprendizaje (MINED, 2008). Dentro del documento se afirma que, la asignatura de Estudios Sociales, en Educación Básica y Media, debe estar orientada a la construcción y consolidación de tres competencias principales: análisis de la problemática social; investigación de la realidad social; y la participación crítica y responsable en la sociedad.

La formación pedagógica de quienes enseñan Ciencias Sociales es otro factor que influye y determina el proceso de enseñanza-aprendizaje. Por ejemplo, para muchos profesores su propia experiencia como estudiantes condiciona su forma de ejercer la docencia. Para el caso, Fernández (citado por Fontán, 2005) afirma que los profesores no aplican los métodos que les enseñaron, sino aquellos que les han aplicado durante su periodo de formación. Por su parte, González (1990) defiende el hecho que “...el profesor puede y debe encontrar la manera de dejar de hacer lo que con él hicieron para comenzar a hacer lo que hubiera querido que con él hubieran hecho" (p. 40).

Sin duda, ante esta realidad emergen nuevos desafíos en la formación de educadores, fente a la integración de las nuevas metodologías, las tecnologías de la información y comunicación (TIC) y las tecnologías del aprendizaje y conocimiento (TAC). Según Morales (2016), en el contexto educativo, las herramientas digitales son:

Todos aquellos software o programas intangibles que se encuentran en las computadoras o dispositivo, que pueden ayudar a interactuar más con la tecnología de hoy en día. Nos ayudan a comunicarnos; a abrir competencias y habilidades en los estudiantes para ser utilizadas en la educación. Además de ser un apoyo en el aprendizaje, también da paso a la innovación de una búsqueda hacia mejores manejos sobre estos materiales (p. 3). 
Tomando en consideración la realidad de las nuevas generaciones que llegan a las aulas universitarias, es evidente que estas necesitan algo que despierte su interés, las estimule y favorezca no solo la memorización de contenidos, sino también la producción de ideas; la reflexión, el pensamiento crítico y la participación activa. Debido a esto es necesario llevar a cabo diferentes estrategias y técnicas activas que propicien el trabajo y el desempeño efectivo en los estudiantes.

Partiendo de esto, surgen las interrogantes: ¿De qué manera se puede incrementar la participación de los estudiantes en el proceso de construcción y consolidación de sus conocimientos? ¿Es posible integral los dispositivos móviles en el aula para darles un uso pedagógico? ¿La aplicación de herramientas tecnológi- cas favorecería de alguna manera los procesos de aprendizaje en los estudiantes?

\section{Metodología}

La investigación-acción tuvo la participación de 24 estudiantes que cursaban la asignatura de Desarrollo Curricular de Ciencias Sociales I, correspondiente a la carrera de Licenciatura en Ciencias de la Educación con especialidad en Educación Básica de la Facultad de Ciencias y Humanidades de la Universidad Católica de El Salvador, durante el ciclo I-2019

Para la recopilación de información se utilizaron diferentes técnicas e instrumentos (ver figura 1). Primero se realizó un diagnóstico durante el primer periodo del ciclo académico I-2019, específicamente durante la primera y segunda semana

\begin{tabular}{|l|r|}
\hline \multicolumn{1}{|c|}{ Técnica empleada } & $\begin{array}{r}\text { Instrumento } \\
\text { utilizado }\end{array}$ \\
\hline $\begin{array}{l}\text { Observación: Empleada en todas las intervenciones didácticas del proceso. } \\
\text { Ayudó a determinar actitudes y conductas de los estudiantes (seguimiento } \\
\text { de indicaciones, productos elaborados, participación en el aula, colabora- } \\
\text { ción entre pares, entre otros). }\end{array}$ & Lista de cotejo \\
\hline $\begin{array}{l}\text { Entrevista grupal: Se realizó después de aplicar cada herramienta digital } \\
\text { (de forma individual o colaborativa). Se evaluaron elementos como: calidad } \\
\text { del trabajo realizado, efectividad y manejo de la herramienta; valoración del } \\
\text { propio proceso de aprendizaje, etc. }\end{array}$ & Guía de entrevista \\
\hline $\begin{array}{l}\text { Encuesta: Se aplicó para valorar el trabajo colaborativo, tomando en cuenta } \\
\text { aspectos como: comunicación, organización del equipo, efectividad en el } \\
\text { manejo del tiempo, recursos y los productos finales. }\end{array}$ & Cuestionario \\
\hline $\begin{array}{l}\text { Diálogo como actividad pedagógica reflexiva: Se ejecutó a lo largo del } \\
\text { proceso investigativo. De manera espontánea se hablaba con los estudiantes } \\
\text { sobre sus impresiones de las herramientas y procesos aplicados. }\end{array}$ & Libreta de registro \\
\hline
\end{tabular}

Figura 1. Descripción de técnicas e instrumentos. 
del mes de febrero. Posteriormente, se realizó la primera intervención del plan de acción durante el segundo periodo del ciclo, mediante la aplicación de estrategias didácticas (herramientas digitales, trabajo colaborativo y seguimiento constante). Finalmente, se hizo la reestructuración del plan de acción para el tercer periodo, durante el mes de mayo e inicios de junio. Acá se ejecutaron las estrategias de: aplicación de herramientas digitales, trabajo colaborativo y trabajo autogestionado o autoaprendizaje.

Las herramientas de tecnología educativa implementadas fueron GoConqr, Proprofs, Padlet, Piktochart y Softwares para crear presentaciones. En total se aplicaron dos intervenciones (cada una de ellas tuvo tres estrategias) durante el ciclo que se desarrolló la asignatura. Algunas estrategias fueron conservadas en ambas; otras se modificaron según las necesidades y los resultados obtenidos durante el proceso. Durante todo el proceso de aplicación se realizó una reflexión constante, considerando la primera y segunda intervención; comparando los aspectos positivos y negativos de cada una.

\section{Resultados}

\section{a. Primera intervención}

De acuerdo con la línea de base en este primer momento de aplicación, se abordaron aspectos relacionados al aumento de la participación activa en clase, la motivación de los estudiantes hacia la asignatura y el fomento de las actitudes de apertura y aprendizaje de los contenidos de las Ciencias Sociales. Se pre- tendía mejorar el promedio de las calificaciones de todo el grupo de estudiantes mediante tres estrategias de trabajo:

\begin{tabular}{|l|l|}
\hline \multicolumn{1}{|c|}{ Estrategias } & \multicolumn{1}{c|}{ Componentes } \\
\hline $\begin{array}{l}\text { Incorporación de } \\
\text { herramientas digitales }\end{array}$ & - GoConqr \\
& - Padlet \\
\hline \multirow{2}{*}{ Trabajo colaborativo } & - Trabajos en el aula \\
\hline \multirow{2}{*}{ Seguimiento } & - Trabajos ex aula \\
\hline continuo & - Doros \\
& - Entrélogos \\
& - Chat en línea \\
\hline
\end{tabular}

Figura 2. Estrategias aplicadas en la primera intervención y sus componentes.

\section{Incorporación de herramientas digitales}

Al incorporar los elementos tecnológicos, los estudiantes se sintieron más interesados en realizar sus actividades; y avanzaron de mejor manera en la adquisición de conocimientos, siguiendo un patrón a su propio ritmo. Fueron ellos quienes organizaron la ruta a seguir para aplicar las actividades y entregar sus productos al final.

Los estudiantes utilizaron sus dispositivos móviles y computadoras personales dentro del aula; también hicieron uso del laboratorio de informática de la Universidad para elaborar productos relacionados con lo visto en clase (ver figura 3).

Las tres herramientas implementadas permitieron integrar dos estrategias: la incorporación de equipos digitales y el trabajo colaborativo simultáneo, 


\begin{tabular}{|c|c|c|}
\hline & Padlet & Proprofs \\
\hline $\begin{array}{l}\text { Es un entorno de estudio en } \\
\text { línea y gratuito. Incluye herra- } \\
\text { mientas de aprendizaje que per- } \\
\text { miten crear, compartir y des- } \\
\text { cubrir mapas mentales, fichas } \\
\text { de estudio, apuntes en línea y } \\
\text { exámenes. También se puede } \\
\text { planificar el estudio y monito- } \\
\text { rizar su proceso en tiempo real. } \\
\text { Permite conectar con amigos y } \\
\text { compañeros para compartir re- } \\
\text { cursos y conocimiento. }\end{array}$ & $\begin{array}{l}\text { Es un mural o póster interac- } \\
\text { tivo que permite publicar, al- } \\
\text { macenar y compartir recursos } \\
\text { multimedia e informaciones } \\
\text { de diferentes fuentes, de ma- } \\
\text { nera individual o en colabora- } \\
\text { ción con un grupo de personas. } \\
\text { Puede utilizarse tanto para pro- } \\
\text { poner actividades en el aula, o } \\
\text { bien para educación en línea, ya } \\
\text { que se puede compartir el Pad- } \\
\text { let en cualquier entorno virtual. }\end{array}$ & $\begin{array}{l}\text { Es una plataforma web que per- } \\
\text { mite la elaboración de recursos } \\
\text { variados. Este software permite } \\
\text { la creación de recursos varia- } \\
\text { dos. Este software permite la } \\
\text { creación de exámenes, cues- } \\
\text { tionarios, tarjetas o flashcards, } \\
\text { juegos como ahorcado, scrub- } \\
\text { ble, crucigramas, rompecabezas, } \\
\text { entre otros, que hacen el aprendi- } \\
\text { zaje más fácil y ameno. }\end{array}$ \\
\hline
\end{tabular}

Figura 3. Descripción de las herramientas implementadas en la primera intervención.

Fuente: Cf. Pontificia Universidad Católica de Valparaíso (s.f.); Fandom (2018) y Baquía (2013).

elaborando mapas mentales, fichas, laboratorios en línea, murales reflexivos, blogs, crucigramas y sopas de letras (ver figuras 4, 5 y 6).

Durante la aplicación de la estrategia se evaluaron dos puntos no favorables, expresados por los estudiantes: la efectividad de la herramienta se vio afectada por la calidad del internet dentro de las instalaciones institucionales, y por la disponibilidad de los centros de cómputo; en segundo lugar, algunos participantes mostraron apatía y distracción en el aula cuando se trabajó con los recursos tecnológicos. Esto motivó el cambio de herramientas para la segunda intervención.

Se tomaron en consideración las sugerencias de los estudiantes sobre actividades que relaciona- ran herramientas que permitieran trabajar con cuadros, pictografías, gráficos o resúmenes de información que favorecieran la creatividad.

\section{Trabajo colaborativo}

A partir del uso de tecnologías de forma colaborativa, los estudiantes se integraron más en los procesos del aula; es decir, cada vez fueron menos los que se sintieron excluidos y aprendieron a valorar el trabajo entre pares. La aplicación de esta estrategia se basó en la elaboración de recursos de manera colectiva; también, intercambio de exámenes aplicando coevaluaciones de los productos. Se elaboraron fichas virtuales de estudio en parejas, mapas mentales, juegos mentales y se valoraron los elemen- 


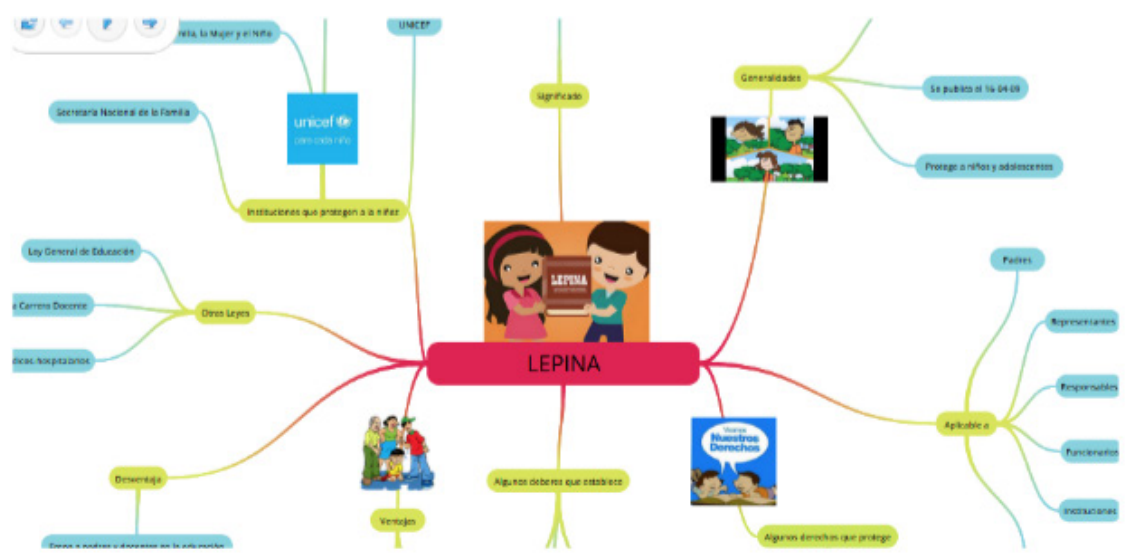

Figura 4. Ejemplo de mapa mental elaborado en GoConqr por una estudiante del grupo.

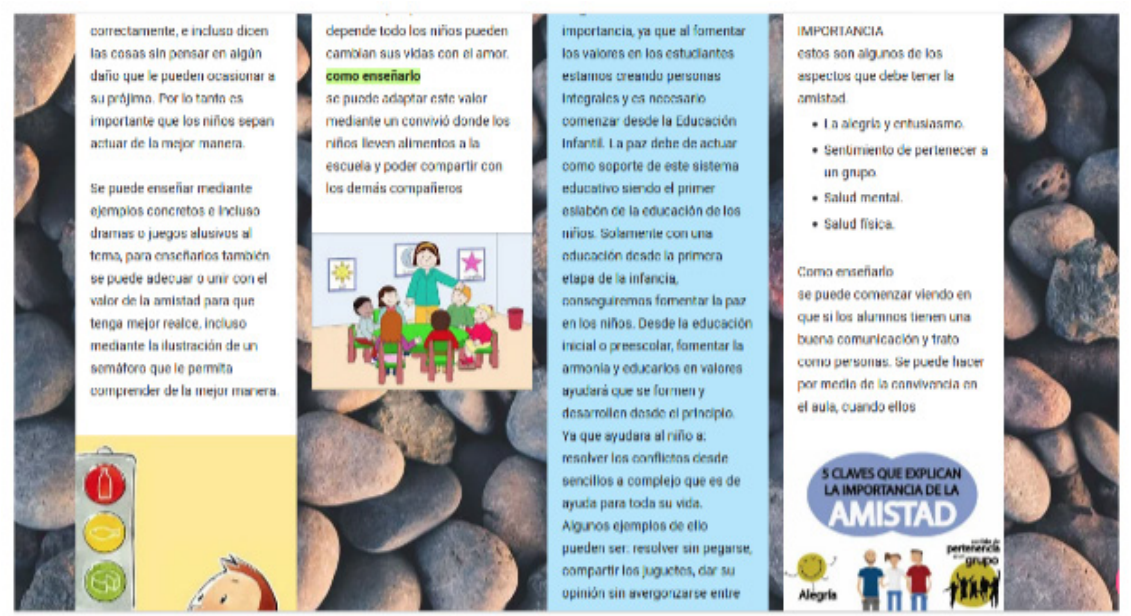

Figura 5. Fragmento de Padlet elaborado de manera colaborativa por los estudiantes.

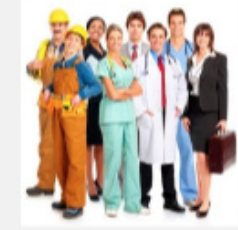

TRABAJO

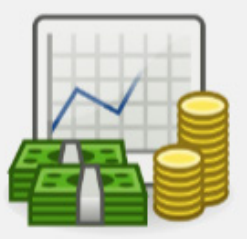

¿Qué es la Economía?
Es todo tipo de acción realizada por el hombre independientemente de sus características o circunstancias.

Ciencia que estudia los métodos más eficaces para satisfacer las necesidades humanas materiales, mediante el empleo de blenes escasos.

Figura 6. Ejemplo de flashcards hechas en Proprofs por una estudiante. 
tos de otros equipos, mediante la escritura de reflexiones (ver figura 7).

A partir de los datos obtenidos, el $83.3 \%$ de los estudiantes consideraron que su aprendizaje se vio favorecido al compartir la información con sus compañeros durante la clase, a través de discusiones presenciales o virtuales. Por otro lado, el 16.7\% restante consideró lo contrario. Una de las limitantes manifestada fue que, aunque el trabajo colaborativo ayudó a desarrollar y comprender mejor el contenido, para algunos también fue fuente de distracción, impidiéndoles rendir con la misma eficiencia que tenían a nivel individual.

\section{Seguimiento constante}

El seguimiento, monitoreo y progreso del proceso de los estudiantes se realizó por medio de foros, chats, diálogos y entrevistas grupales para conocer su opinión respecto a las herramientas. Expresaron que estas eran creativas, de fácil manejo y de ayuda para la asimilación de los contenidos, además de tener una interfaz amigable. Un aspecto negativo fue el hecho que solo pueden utilizarse en línea. En cuanto al desarrollo de la clase, se logró que ellos se involucraran más en el proceso de aprendizaje, pues muchas veces es muy tedioso escuchar solamente al docente que está impartiendo el contenido.

\section{b. Segunda intervención}

A partir del análisis de los resultados, y tomando en cuenta las opiniones de los estudiantes, se efectuaron modificaciones en el plan de acción para la segunda intervención (ver figuras 8 y 9).

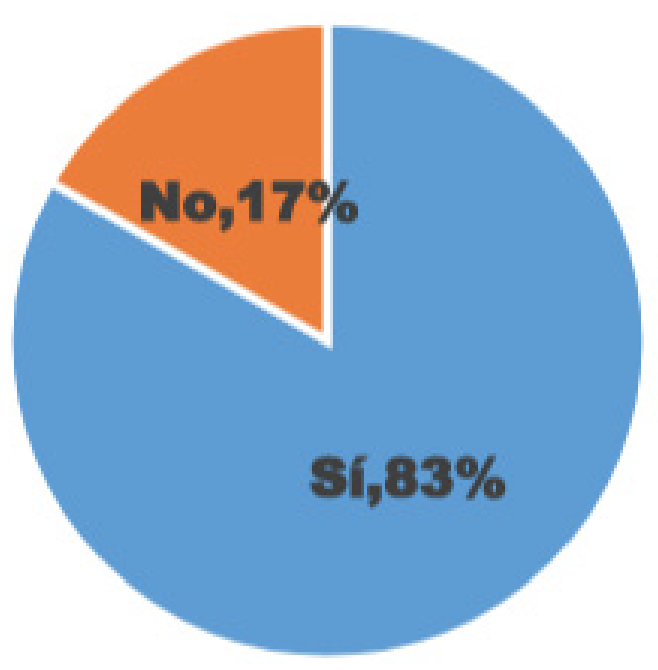

Figura 7. Favorecimiento del trabajo colaborativo en el aprendizaje. 


\begin{tabular}{|l|l|}
\hline \multicolumn{1}{|c|}{ Estrategias } & \multicolumn{1}{c|}{ Componentes } \\
\hline Incorporación de herramientas digitales & $\begin{array}{l}\text { - Softwares en línea para hacer presentaciones } \\
\text { - Piktochart } \\
\text { - Proprofs }\end{array}$ \\
\hline Trabajo colaborativo & - Trabajos ex aula \\
\hline Aprendizaje autogestionado & $\begin{array}{l}\text { - Exposición y socialización de contenidos } \\
\text { - Portafolios de aprendizaje }\end{array}$ \\
\hline
\end{tabular}

Figura 8. Estrategias aplicadas en la segunda intervención y sus componentes

\begin{tabular}{|c|c|c|}
\hline $\begin{array}{c}\text { Softwares en línea para } \\
\text { hacer presentaciones }\end{array}$ & Piktochart & Proprofs \\
\hline $\begin{array}{l}\text { Este tipo de programas per- } \\
\text { miten crear presentaciones } \\
\text { dinámicas de manera clara y } \\
\text { sencilla. Poseen una gran di- } \\
\text { versidad de plantillas predefi- } \\
\text { nidas, que facilitan la edición } \\
\text { y personalización de la infor- } \\
\text { mación. Permite incorporar } \\
\text { gráficos, imágenes, texto y } \\
\text { otros recursos de apoyo. } \\
\text { Las herramientas admiten su } \\
\text { uso en cualquier dispositivo } \\
\text { con conexión a internet. }\end{array}$ & $\begin{array}{l}\text { Es una herramienta en línea que } \\
\text { permite crear infografías gratui- } \\
\text { tamente. Esta ofrece plantillas } \\
\text { predefinidas en las cuales es } \\
\text { posible agregar y dar formato } \\
\text { al texto (tamaño, tipo, color, ali- } \\
\text { neación, etc.); agregar conteni- } \\
\text { dos multimedia como imágenes } \\
\text { y videos, entre otras posibilida- } \\
\text { des. En resumen, el creador de } \\
\text { la infografia puede ordenar la } \\
\text { presentación de su tema. }\end{array}$ & $\begin{array}{l}\text { Es una plataforma web que } \\
\text { permite la elaboración de re- } \\
\text { cursos variados. Este software } \\
\text { permite la creación de exáme- } \\
\text { nes, cuestionarios, tarjetas o } \\
\text { flashcards, juegos como ahor- } \\
\text { cado, scrubble, crucigramas, } \\
\text { rompecabezas, entre otros, que } \\
\text { hacen el aprendizaje más fácil } \\
\text { y ameno. }\end{array}$ \\
\hline
\end{tabular}

Figura 9. Descripción de las herramientas aplicadas en la segunda intervención.

Fuente: Cfr. Pontificia Universidad Católica de Valparaíso (s.f.) y Aulafácil (2019)

\section{Incorporación de herramientas de aprendizaje}

Atendiendo a las sugerencias de los estudiantes, en la segunda intervención se utilizaron herramientas digitales para la elaboración de productos gráficos, con la finalidad de asimilar la información de manera visual y aplicar habilidades creativas.
Los aspectos que se evaluaron en estas estrategias fueron: funcionalidad de la herramienta; utilidad para elaborar productos en línea; facilidad para compartir el trabajo con otros en redes sociales, correos, enlaces o descargas; y uso de las opciones de trabajo disponibles (texto, formas, colores, etc., inserción de imágenes y elementos multimedia). 
Para evaluar las herramientas digitales se diseñó una escala de apreciación, en donde: $1=$ No fue posible trabajar efectivamente; $2=$ Se logró trabajar, aunque con algunos problemas, y $3=$ Se trabajó efectivamente, sin problemas.

Con base en ella, los estudiantes asignaron a las herramientas utilizadas una nota promedio de 2.6 en funcionalidad. También afirmaron su conformidad con la facilidad para elaborar los diferentes trabajos solicitados durante el ciclo de intervención, destacando la variedad de fuentes y opciones para evidenciar la creatividad e innovación en los productos elaborados. Otro de los aspectos que más valoraron los estudiantes fue poder descargar sus productos en PDF o en formato de imagen, permitiéndoles la portabilidad del trabajo realizado, sin necesidad de tener acceso a internet. Gracias a ello pudieron compartir sus resultados en redes sociales y mediante la generación de enlaces.

La limitante que estas herramientas presentaron fue su exclusividad para trabajarlas en línea; no obstante, este problema se vio menguado con el trabajo desde casa o fuera del aula en sitios donde lo permitiese la conexión.

\section{Trabajo colaborativo}

Esta vez, la modalidad del trabajo colaborativo fue en su mayoría ex aula ${ }^{1}$, dejando las sesiones presenciales para acordar aspectos generales de las actividades, la distribución de tareas entre los integrantes y el establecimiento de reglas de trabajo al interior de cada uno de los equipos.

Los estudiantes pusieron en práctica las habilidades de delegación de roles y funciones para la organización de las socializaciones que debían desarrollar en clase. Si bien las tareas fueron divididas, los estudiantes manifestaron estar atentos ante las dificultades que los compañeros pudieran presentar. De esta manera, los miembros del equipo se auxiliaron oportunamente entre sí.

Para la elaboración de los portafolios digitales, diez de las doce parejas manifestaron que no tuvieron mayor problema en la realización de las actividades; sin embargo, los dos restantes tuvieron dificultades de comunicación y organización, por lo que no entregaron sus portafolios completos. Una de estas duplas entregó portafolios de manera individual.

\section{Aprendizaje autogestionado}

Los participantes se mostraron entusiasmados al preparar por sí mismos los contenidos, y mostrarlos a sus compañeros a través de medios alternos, como presentaciones e infografías. De esta forma, tomaron ellos el rol de facilitadores del aprendizaje, dejando a un lado la figura del docente como único transmisor del conocimiento. Al mismo tiempo, los estudiantes fueron capaces de desarrollar actitudes de responsabilidad ante la entrega de las dife-

\footnotetext{
1. Término utilizado en lenguaje pedagógico para referirse a todas las acciones que se llevan a cabo fuera del aula de clase, encaminadas al refuerzo y fortalecimiento del proceso de aprendizaje de los estudiantes.
} 
rentes actividades en tiempo y forma: cumplir con las funciones asignadas, realizar búsquedas bibliográficas acertadas y búsqueda de técnicas para facilitar la información en la socialización con sus compañeros.

La autorregulación del aprendizaje permitió continuar con el modelo self-paced. Dentro de este, los estudiantes son los únicos responsables de llevar a cabo las diferentes actividades, tanto la organización y socialización de una intervención didáctica, como la elaboración de un portafolio digital con productos relacionados a la construcción, y consolidación de los aprendizajes obtenidos.

\section{Discusión}

En los preámbulos de la filosofía del Espacio Europeo de Educación Superior (EEES), Huber (2008) citado por Iglesias, Lozano y Martínez (2012), describió los vínculos teóricos más relevantes que implican la enseñanza y el aprendizaje activo en las universidades. Él expresa que "(...) la participación de los estudiantes en su aprendizaje está orientada hacia cinco rasgos fundamentales: aprendizaje activo, aprendizaje autorregulado, aprendizaje constructivo, aprendizaje situado y aprendizaje social (...)" (p. 4). En un mundo caracterizado por la incidencia masiva de la sociedad de la información y las tecnologías de información y comunicación, es menester aplicar estas nuevas herramientas en las aulas de clase; no solo a nivel de Educación Básica, sino también nivel Superior. Es decir, la responsabilidad de reestructurar las metodo- logías de enseñanza-aprendizaje, a propósito de orientar procesos más activos de formación en los estudiantes universitarios.

Hernández y Peñalosa (2015) lo afirman en su investigación:

Las tecnologías de la información y la comunicación forman parte de un nuevo paradigma tecnológico que vive la humanidad y que trasciende a la educación, pues plantea un impacto social global. Evidentemente, la educación es un área en la que estas herramientas tienen impacto. Dichas tecnologías se han incorporado gradualmente en todos los niveles educativos, por lo que ya es tiempo de dar testimonio de las formas en que se utilizan en el aula, así como su verdadera repercusión en el aprendizaje (p. 15).

De modo que se propicien “(...) nuevas maneras de trabajar, de comunicarnos, de relacionarnos, de aprender, de pensar y, en suma, de vivir" (Coll y Monereo, 2008, p. 19).

Durante la aplicación de estas herramientas digitales, en conjunto con estrategias como trabajo colaborativo, seguimiento constante y trabajo autogestionado, los beneficios en el proceso de construcción y consolidación de enseñanza-aprendizaje de los estudiantes han sido evidentes.

Asimismo, es importante recalcar que la aplicación de esta nueva metodología y la elaboración de nuevas actividades también sirvió como una forma diferente y activa de evaluar a 
los aprendices. Al considerar el alternative assessment o evaluación alternativa, propuesta en los nuevos modelos educativos, que garantiza mejores resultados dentro del proceso de enseñanza-aprendizaje; y que se vieron reflejados cualitativamente en la nula reprobación de los estudiantes del grupo de estudio.

De esta manera se garantizó un proceso formativo activo. Además, se pusieron en juego habilidades como: la organización del tiempo, responsabilidad, delegación de funciones; toma de decisiones, resolución de problemas, comunicación efectiva, trabajo en equipo, entre otras. Todas estas forman parte del listado de habilidades que son necesarias para el siglo XXI.

Esto quiere decir que la integración de las herramientas tecnológicas educativas, en combinación con otras estrategias efectivas de trabajo, permite abrir una nueva perspectiva no solo en el proceso de construcción de aprendizaje de esta asignatura, sino también en los mecanismos de evaluación de la misma. Esto descarta el paradigma que Estudios Sociales se limita a la memorización de datos y fechas, y que únicamente puede evaluarse mediante pruebas estandarizadas.

A partir de este estudio, se ha puesto en práctica una nueva alternativa de evaluación aplicada a esta rama del saber. Con ello se brinda a los colegas y lectores una forma diferente para determinar no solo el aprendizaje conceptual, sino un aprendizaje integral en donde el estudiante se desenvuelva en múltiples dimensiones de su personalidad como aprendiz.
El uso de herramientas tecnológicas en el aula debe estar orientado, previamente, con un objetivo de aprendizaje, cuya base debe ser la de mejorar el proceso de enseñanza-aprendizaje y evitar en el máximo nivel que sea una pérdida del tiempo pedagógico. Al establecer las herramientas y estrategias de trabajo es importante mantener el enfoque de aprendizaje activo y significativo, en donde el estudiante sea el protagonista de la construcción y consolidación de su propio aprendizaje. Así, Balderas, Gómez y Hernández (2014) lo afirman “(...) el proceso de inclusión de TIC en las actividades curriculares debe acompañarse de la creatividad para diseñar las actividades por realizar, con el fin de lograr la atención, motivación y participación activa del estudiante en el desarrollo de la clase" (p. 18).

Por otro lado, algo que puede representar una limitante u obstáculo a lo enunciado anteriormente, es la poca disponibilidad y motivación por parte de algunos estudiantes; así como que los procesos seleccionados y acciones a ejecutar no respondan a las necesidades e intereses de los discentes. Esto, en lugar de beneficiar ampliamente el proceso formativo puede ocasionar el efecto contrario. Al mismo tiempo, el uso constante de dispositivos móviles para fines de entretenimiento, enviando mensajes de texto o revisando redes sociales, interfiere significativamente en el desarrollo de las clases y los desvía de los objetivos pedagógicos. 
En respuesta a esto, es importante estar atentos a las conductas de los estudiantes y sus reacciones ante las intervenciones, pues son ellos, mediante sus actitudes, quienes dan la pauta para tomar iniciativas y realizar los cambios pertinentes, que les ayuden a ver el aprendizaje como un proceso activo. Son ellos quienes instan a cuestionar la propia praxis con inte- rrogantes como las siguientes: ¿cómo innovar en el aula? ¿Qué nuevas estrategias se pueden aplicar para que los estudiantes mejoren en sus procesos de aprendizaje? ¿Cuál debe ser el rol del docente frente a las nuevas tecnologías y su potencial en el aula?

\section{Referencias}

Aulafácil (2019). Piktochart. Recuperado de https://www.aulafacil.com/cursos/diseno-grafico-cad/infografia/que-es-piktochart-130970

Aventura Amazonia (2017). Actividades extraescolares. Definición y tipos. Recuperado de https://www.aventura-amazonia.com/nos-gusta-el-cole/201633

Balderas, M.; Gómez, M. y Hernández, C. (2014). Inclusión de las tecnologías para facilitar los procesos de enseñanza-aprendizaje en ciencias naturales. Revista Electrónica Actualidades Investigativas en Educación, 14(3), 1-19. Recuperado de https://www.redalyc.org/ pdf/447/44732048010.pdf

Baquía (2013). Proprofs, una nueva opción para cursos y formación online. Recuperado de https://www.baquia.com/emprendedores/2013-04-29-proprofs-una-nueva-opcion-para-cursos-y-formacion-online

Coll, C. y Monereo, C. (2008). Educación y aprendizaje en el siglo XXI: nuevas herramientas, nuevos escenarios y nuevas finalidades. Madrid. Recuperado de https://www.salesianoscentroamerica.org/component/easyfolderlistingpro/?view $=$ download $\&$ format $=$ raw $\&$ data $=\mathrm{eNp}$ FUF1PwjAU_Ss3fTfbJKKUpwnFjCAjCAn4Qi7bZdR069J2SGL873YbxKf2nN7zcYs8iviP5UPOTlr1ZNjY8tGAM11iQTZ41UcltaMMgxVapw2qw7y5UCVVMNOmxEzqCmo0CKRgY_CIXzp4DKMBPICosoYqZzQYKvwcKsgJZqtNG-JzWWPJ9LG2ozgL709FQ9b9V3rm7HDouBa99NIKS2phyF17DHtW5mwsediLDKka3blzfeIseI83 Yp3EC4hX6T69eZ2korvXwGMx3U7iSZIuYe_n1mI5TT7juVwwsQSxgI_kbZHCbtdNc0ZXd7vV-elWla61NGTvPVwiXxCdw-xcXPrvYONjx468wtBF0ne_oq9faF0oX-P3DywCf3Q

Fandom (2018). Herramientas colaborativas en educación: GoConqr. Recuperado de https://herramientas-colaborativas-en-educacion-by-eimy.fandom.com/es/wiki/GoConqr 
Fontán, T. (2005). La formación docente para el uso de las tecnologías de la información y comunicación. El Guiniguada, 14, 103-118. Recuperado de https://accedacris.ulpgc.es/bitstre am/10553/5783/1/0235347_00014_0008.pdf

González, F. (1990). Sobre la situación y el significado de la didáctica. Revista Complutense de Educación, 1(1), 31-54. Recuperado de https://revistas.ucm.es/index.php/RCED/article/ view/RCED9090130031A

Hernández, G. y Peñalosa, E. (2015). Las tecnologías digitales como herramientas de enseñanza-aprendizaje en la UAM Cuajimalpa. En Innovación educativa y apropiación tecnológica: experiencias docentes con el uso de las TIC (pp. 15-28). México: Universidad Autónoma Metropolitana. Recuperado de http://www.cua.uam.mx/pdfs/biblioteca/colecciondelibros-uamc/ pdfs/08innovacion_educativa.pdf

Iglesias, M.; Lozano, I. y Martínez, M. (2012). La utilización de herramientas digitales en el desarrollo del aprendizaje colaborativo: análisis de una experiencia en Educación Superior. Revista de Docencia Universitaria, 11(2) Universidad de Alicante. España. https://doi. org/10.4995/redu.2013.5579

Ministerio de Educación de El Salvador (2008). Currículo al Servicio del Aprendizaje. (2 ${ }^{\mathrm{a}}$ ed.) San Salvador. Recuperado de https://www.oei.es/historico/pdfs/curriculo_aprendizaje_salvador.pdf.

Morales, G. (2016). Herramientas digitales [presentación de diapositivas]. Slideshare. Recuperado de https://es.slideshare.net/gabrielamorales56/herramientas-digitales-60428473

Ortega, D. (2014). La enseñanza de las ciencias sociales, las TIC y el Tratamiento de la Información y Competencia Digital (TICD) en el grado de Maestro/a de Educación Primaria de las Universidades de Castilla y León. Enseñanza de las ciencias sociales: revista de investigación, 14, 121-134. Recuperado de https://www.redalyc.org/html/3241/324148872012/

Pontificia Universidad Católica de Valparaíso (s.f). Emaze. Recuperado de: http://recursostic.ucv. cl/wordpress/index.php/essential_grid/emaze/

Pontificia Universidad Católica de Valparaíso (s.f). Padlet. Recuperado de: http://recursostic.ucv. cl/wordpress/index.php/essential_grid/padlet-perfil-docente/

Sánchez, P. (1992). Sobre la enseñanza en el área de Ciencias Sociales. En La enseñanza de la Historia, la Geografía y las Ciencias Sociales (pp. 40-52) Madrid, España: Editorial Complutense. 